\title{
Lymphocytic tumours of the conjunctiva
}

\author{
GWYN MORGAN
}

From the Department of Pathology, Institute of Ophthalmology, London

SYNOPSIS Twenty-six cases of lymphocytic tumour of the conjunctiva, which were originally classified as benign lymphoma and lymphosarcoma, were followed up for more than five years, They were then reclassified into non-disseminating and disseminating groups. Only when germinal follicles are present can a histological diagnosis of benign lymphoma be made. Moreover, it is only when lymphoblasts are seen to be infiltrating the tissues that a definitive diagnosis of lymphosarcoma can be made. The remaining tumours, which represent the large majority of lesions, show a very similar or identical histological picture, and a diagnosis of benignity or malignancy can only be made after a prolonged follow up. The possible nature of the non-disseminating lymphocytic tumours is briefly discussed.

Lymphocytic tumours of the conjunctiva are usually classified as lymphoma or benign lymphoma, lymphosarcoma, or lymphatic leukaemic deposits. With the exception of the latter, which in most cases can be readily diagnosed haematologically, this classification presents some difficulties because the histological diagnosis on initial biopsy is sometimes belied by the subsequent clinical course. Thus a tumour diagnosed 'benign' may later disseminate and conversely a 'malignant' tumour may not. Either the original diagnosis was in error or there is no sharp histological distinction between benignity and malignancy in this group of tumours.

In order to examine these difficulties and to determine to what extent histological features can be correlated with the subsequent clinical course, I carried out a long-term follow-up of these cases of lymphocytic tumour of the conjunctiva (excluding leukaemic deposits). On the record of their clinical behaviour for a period of more than five years, they were reclassified into non-disseminating and disseminating tumours and the original clinical and histological features reviewed retrospectively in order to discover any features that might have pointed to their subsequent course.

Examples of 'benign lymphoma' have been recorded by many authors. Hochheim (1900) described a woman of 79 years with bilateral tumours. Other cases have been recorded by van Duyse (1905) who was the first worker to treat the lesions by irradiation, Baslini (1907), Cosmettatos (1910), and Coats Received for publication 12 November 1970.
(1915) who described five cases affecting young people. The tumours recorded by Coats were slowly growing and were situated at the inner portion of the conjunctiva, three affecting the plica, one the caruncle, and the fifth affecting the limbus. The lesions were bilateral and symmetrical in one case. Bedell (1922) described the first case in America, and other cases have been described by SpecialePiccichè (1927), Saradarian (1940), Ayoub (1948), Svoboda (1948), Magni (1949), King and Ashton (1949), Blaxter (1955), Friedman, Borrelli, and Geleris (1955), Charamis (1957), Valvo (1959), Scuderi and Cardia (1959), Lerman (1960), TrzcińskaDąbrowska (1963), Fronimopoulos, Kofinas, and Lambrou (1964), and Buiuc, Cordun, Beşchea, Popovici, and Rinea (1966).

Examples of 'lymphosarcoma' of the conjunctiva have been recorded by Vollaro (1899), Werner (1904), Fergus (1905), Goldzieher(1907), and Teulières(1910) who described a lesion near the caruncle which was associated with cervical lymphadenopathy; Coats (1915), Casolino (1916), Bedell (1922), Shannon and McAndrews (1932), Ennema (1935), Heath (1949), Roveda (1949), Heritier and Wachtel (1951), Capalbi (1951), Cometta (1952), Junghannes (1953), Schardt (1956), Offret and Rouher (1956), Hilaris, Katrakis, and Karapatakis (1959), Chatterjee (1959), Vancea, Lazarescu, Cerne, and Vaighel (1959), Lerman (1960), and Theodore (1960) who described a patient with lymphosarcoma of the stomach who developed lesions of both conjunctivae several years later; and by Bernardi, Martuzzi, and Palmieri (1963). The 


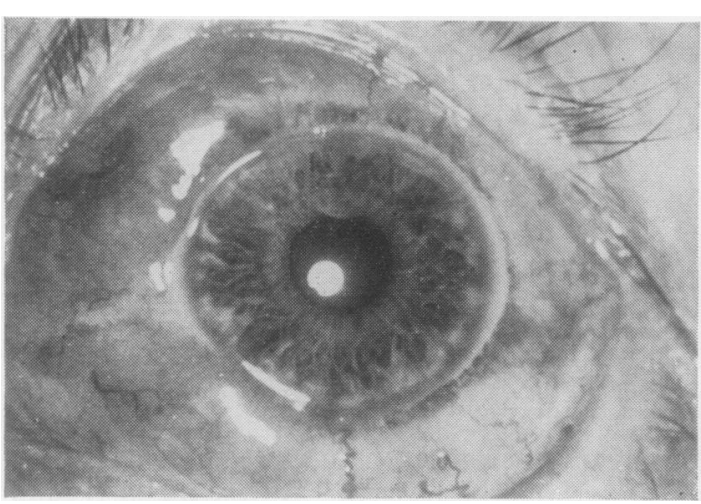

Fig. 1 Clinical photograph of the eye, showing a fleshy lymphocytic tumour of the conjunctiva.

conjunctival lesions may precede, succeed, or be concomitant with lesions elsewhere in the body.

Clinically, lymphocytic tumours of the conjunctiva are typically small, firm, salmon pink-coloured, fleshy, slowly growing, and painless lesions that can be found anywhere in the conjunctiva and may extend onto the cornea or into the orbit. The lesions may be bilateral, occasionally multiple, and usually have a sharply demarcated border, the tumour tending to mould itself to the contour of the surrounding structures (Fig. 1).

The usual histological appearances are of a sheet of lymphocytes lying in a delicate fibrovascular stroma (Fig. 2) usually with occasional plasma cells (Fig. 3) and reticulum cells. Germinal follicles (Fig. 4) are a feature of some cases, and foci of reticulum cells (Fig. 5), multilobulated reticulum cells (Fig. 6), Russell bodies (Fig. 7), epithelioid cells (Fig. 8), and mitotic figures and fibrosis (Fig. 9) may be seen in other tumours. Lymphoblasts may be seen infiltrating the conjunctival tissues (Fig. 10) in some cases.

\section{Material}

Twenty-six cases of lymphocytic tumour of the conjunctiva were studied and followed up for more than five years (Table I). The lesions were classified into benign lymphoma and lymphosarcoma and at the end of five years were classified again into nondisseminating and disseminating lymphocytic tumours. The clinical and histological features were then reviewed in retrospect in order to see whether or not there were any features that might have indicated the final outcome.

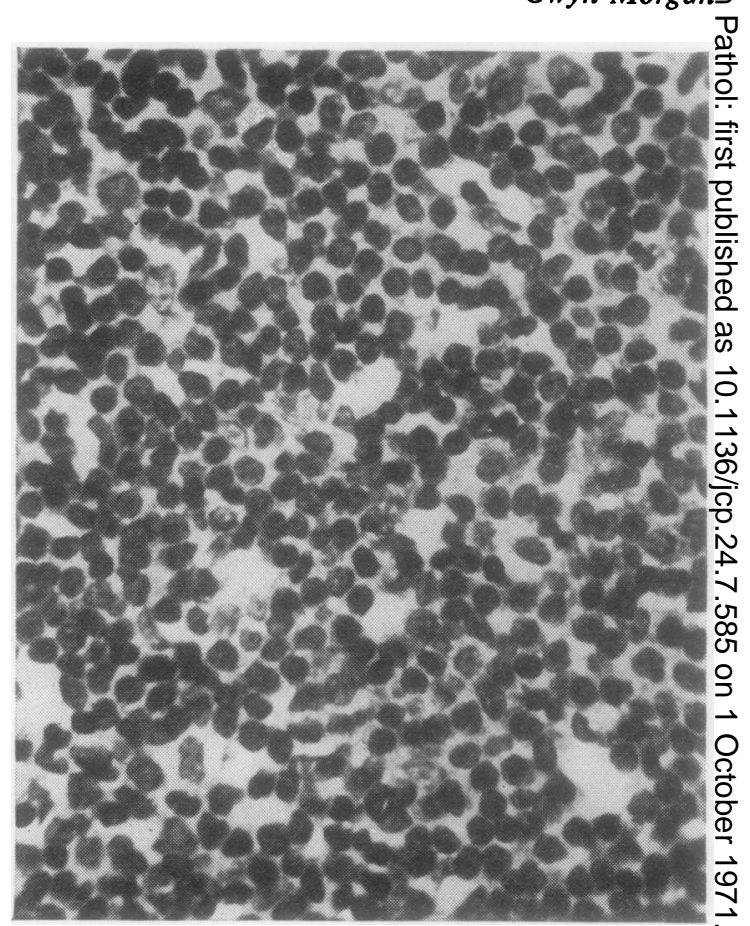

Fig. 2 Lymphocytic tumour of the conjunctiva, composed of a sheet of lymphocytes and occasional reticulum cells. $H$ and $E \times 590$.

\section{Results}

Of the 26 cases followed up, the initial diagnosis was benign lymphoma in 18 cases and lymphosarcoma ino eight cases (Table II). The final diagnosis in the group of benign lymphomas was 16 non-disseminating tumours and two disseminating tumours. Ofô the eight cases diagnosed as lymphosarcoma, four disseminated and four did not (Table III). A review? of the clinical and histological features of the twos cases diagnosed initially as benign lymphoma (cases 14 and 23) and later disseminated as lymphosarcomas is shown in Table IV. A benign lymphoma ${ }^{\mathrm{N}}$ (case 4) which did not disseminate is included for 0 comparison. It will be seen that there were now clinical or histological changes which might haveo suggested that these two tumours would haveo disseminated as lymphosarcomas. Both cases showe histological features that were similar or identicas? with case 4 which did not disseminate, the patiento being alive and well 16 years after the onset. Bothe tumours (cases 14 and 23) showed a sheet of lymphocytes with occasional reticulum cells (Fig. 11) $\stackrel{\mathbb{Q}}{\circ}$

\section{$\frac{1}{2}$}




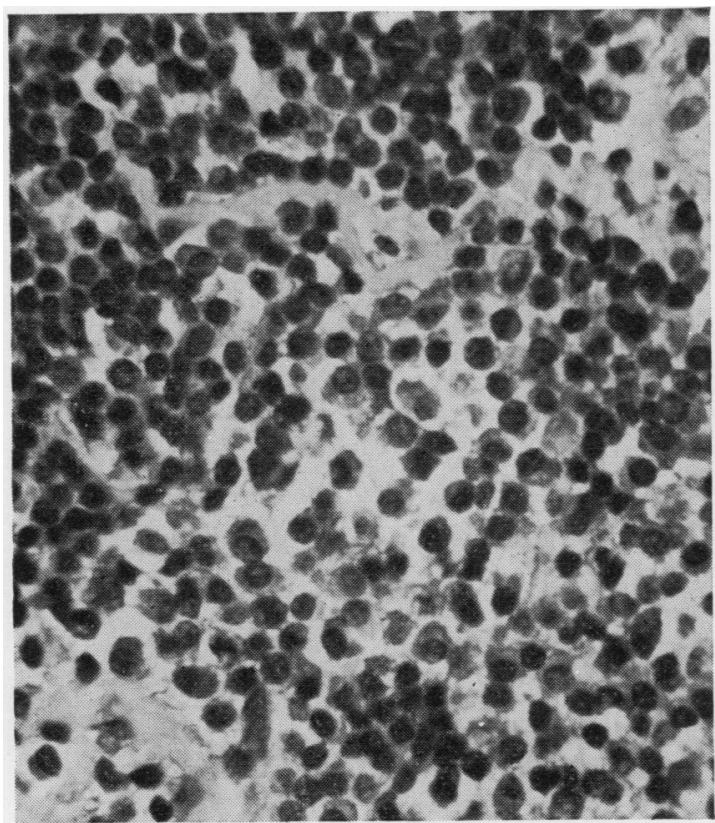

Fig. 3 Lymphocytic tumour of the conjunctiva, showing plasma cells near the centre of the field. $\mathrm{H}$ and $E \times 590$.

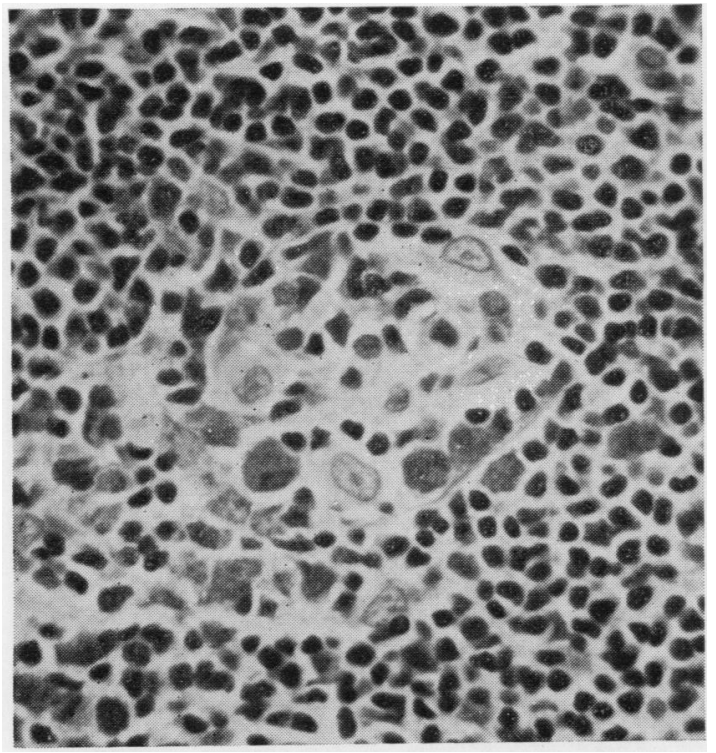

Fig. 5 Lymphocytic tumour of the conjunctiva, showing a focus of reticulum cells. $H$ and $E \times 590$.

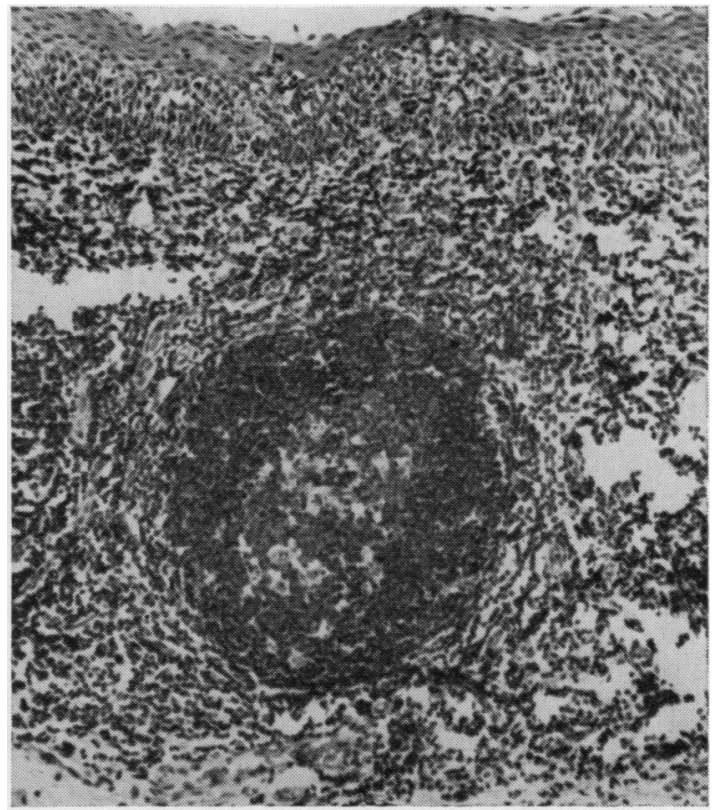

Fig. 4 Lymphocytic tumour of the conjunctiva, showing a germinal follicle. $H$ and $E \times 130$.

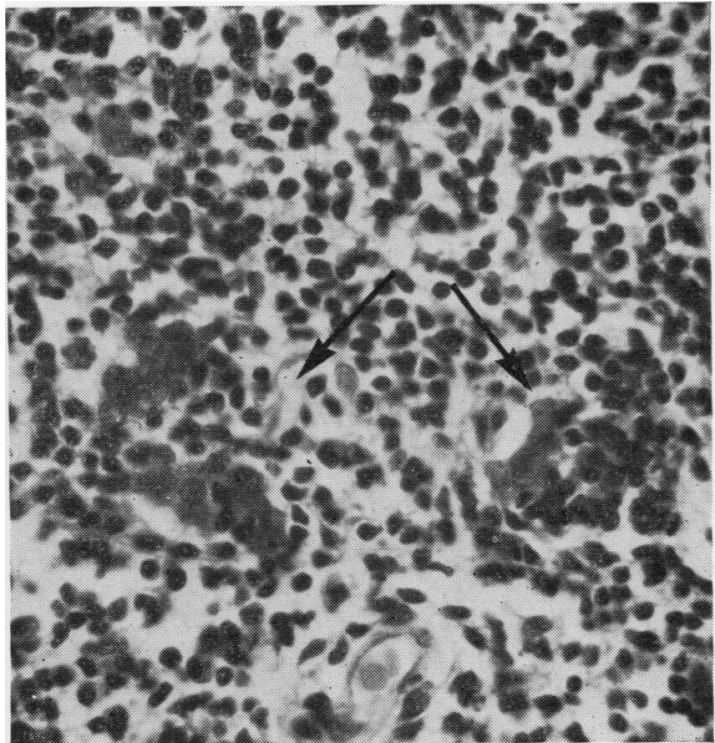

Fig. 6 Lymphocytic tumour of the conjunctiva, showing two multilobulated reticulum cells (arrows). $H$ and $E \times 590$. 


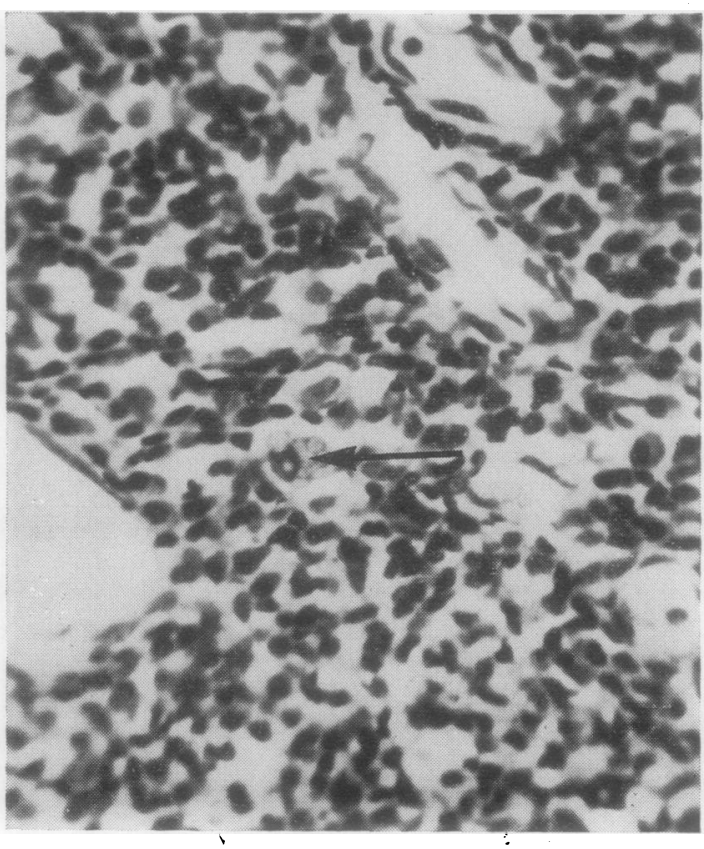

Fig. 7 Lymphocytic tumour of the conjunctiva, showing a Russell body near the centre of the field (arrow). $H$ and $E \times 590$.

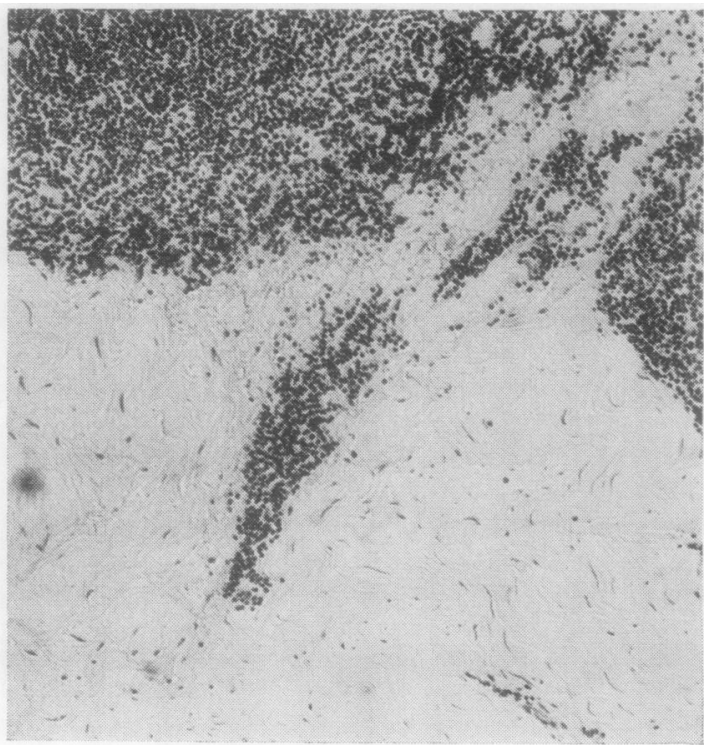

Fig. 9 Lymphocytic tumour of the conjunctiva, showing fibrosis. $\mathrm{H}$ and $\mathrm{E} \times 130$.

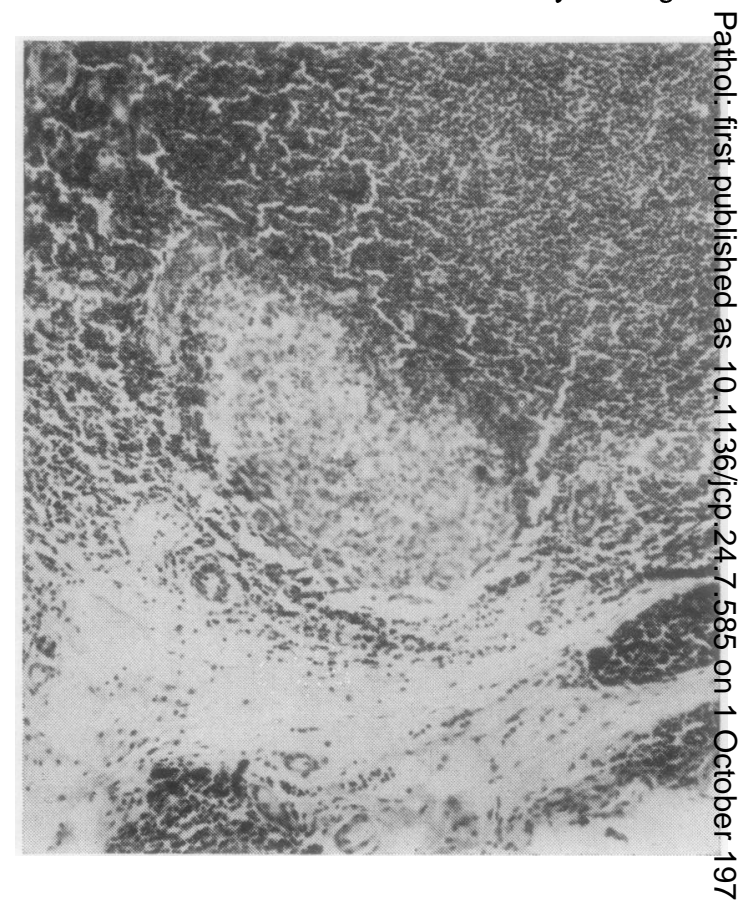

Fig. 8 Lymphocytic tumour of the conjunctiva, showing . a focus of epithelioid cells centrally. $H$ and $E \times 130$.

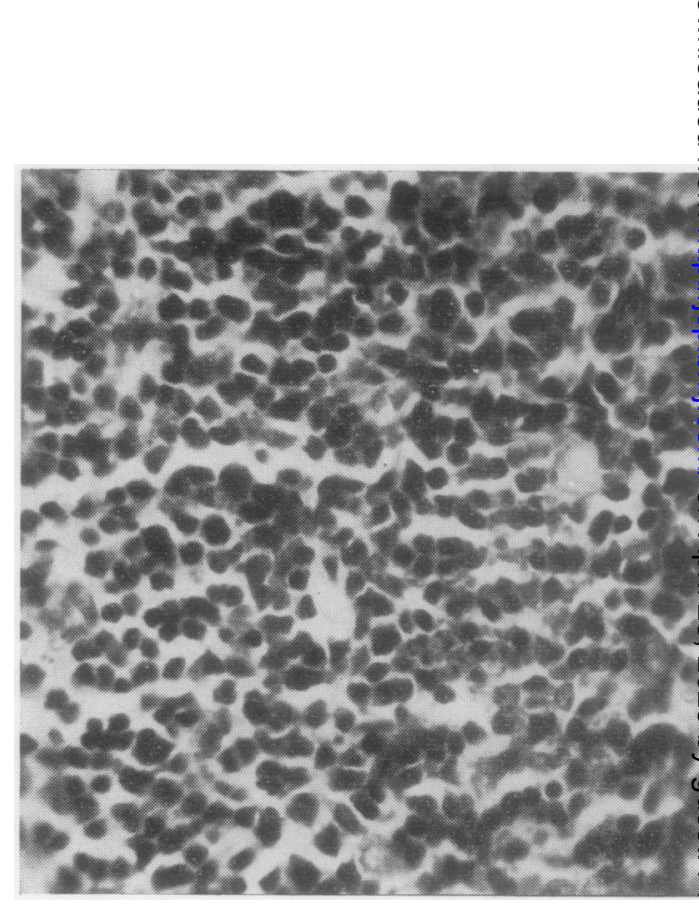

Fig. 10 Lymphocytic tumour (lymphosarcoma) of the

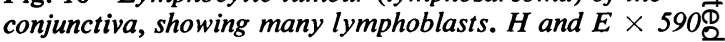

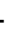

$\overrightarrow{0}$ 然

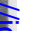




\begin{tabular}{llllllll}
\hline Case Age Sex Site & $\begin{array}{l}\text { Relevant Clinical Gross } \\
\text { Features }\end{array}$ & Histopathology & $\begin{array}{l}\text { Primary } \\
\text { Treatment }\end{array}$ & Recurrence & Follow Up
\end{tabular}

$132 \quad M \quad$ Inner

Inner

Bilateral lesions Localized

(L eye)

for 12 months, raised mass

blood count,

ESR, and WR

normal

2

49 F $\begin{aligned} & \text { Lower } \\ & \text { fornix } \\ & \text { (L eye) }\end{aligned}$

Recent onset

Similar lesion

excised from $\mathbf{R}$

conjunctiva 2

years previously

Localized

raised mass

Fornices Present 1 month Soft, pink,

$3 \quad 63 \quad F$

caruncle Blood count raised mass (R eye) normal
A sheet of lymphocytes Excision

with occasional

reticulum cells

A sheet of lymphocytes Biopsy

with well defined

follicles of reticulum

cells, epithelium

infiltrated

A sheet of lymphocytes Biopsy

with occasional

reticulum cells
No

Alive and well with no lesions 18 years after onset after biopsy with no lesions recurrence 15 years after excised onset

No

Died 11 years after onset from coronary occlusion. No tumour present

No

Alive and well

A sheet of lymphocytes Biopsy and with a few plasma cells irradiation and reticulum cells, (300 r) some of the latter

being multilobulated

A sheet of lymphocytes Biopsy and occasional

reticulum cells
Present 1 month Localized with ptosis and mass

(L eye) chemosis, blood$$
\text { count normal }
$$

raised pinkish$$
\begin{aligned}
& \text { Present } 2 \text { years, Widespread } \\
& \text { gradual increase raised pinkish }
\end{aligned}
$$

$$
\text { retict }
$$




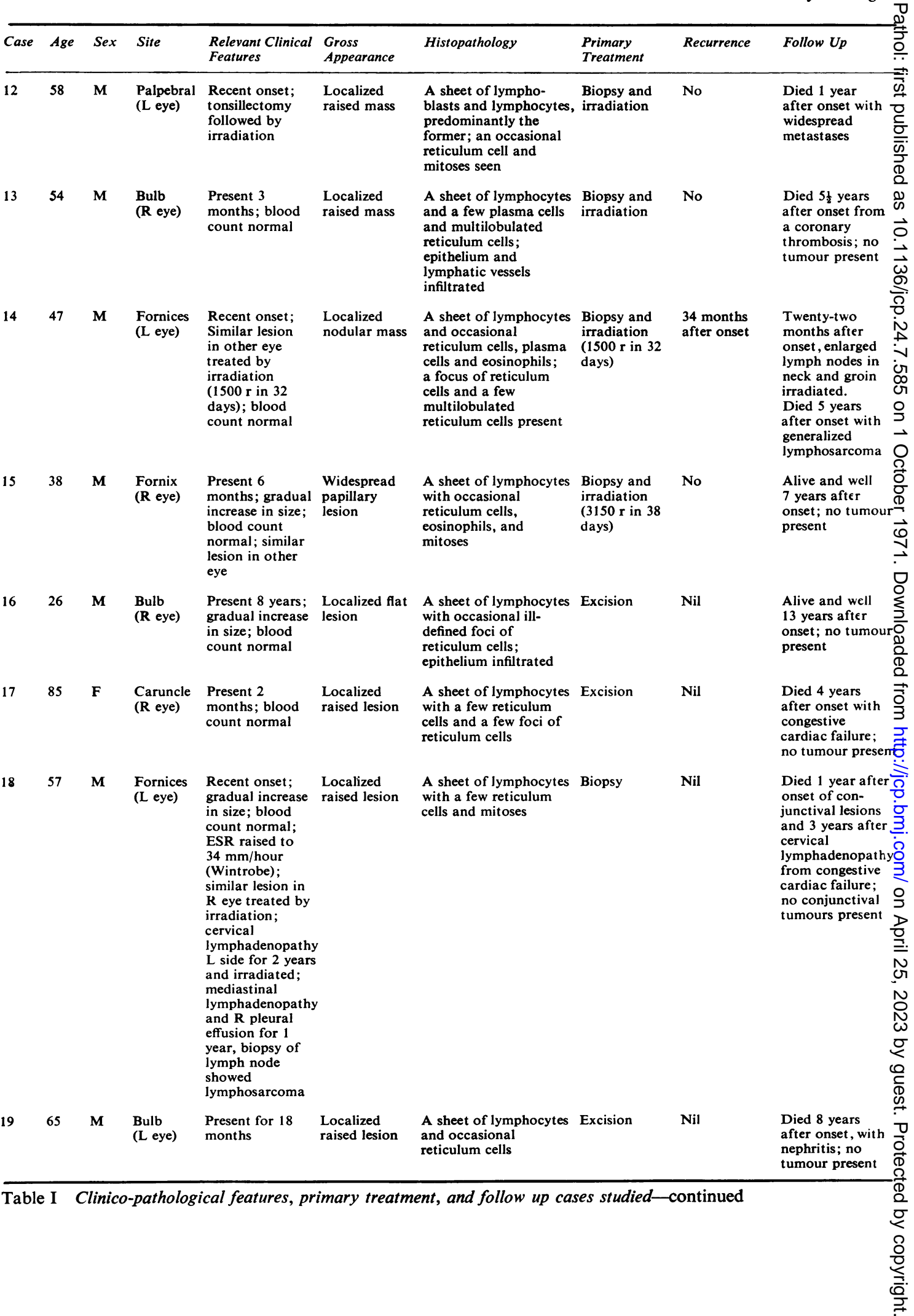




\begin{tabular}{|c|c|c|c|c|c|c|c|c|c|}
\hline Case & Age & $\operatorname{Sex}$ & Site & $\begin{array}{l}\text { Relevant Clinical } \\
\text { Features }\end{array}$ & $\begin{array}{l}\text { Gross } \\
\text { Appearance }\end{array}$ & Histopathology & $\begin{array}{l}\text { Primary } \\
\text { Treatment }\end{array}$ & Recurrence & Follow Up \\
\hline 20 & 69 & $\mathbf{M}$ & $\begin{array}{l}\text { Lower } \\
\text { fornix } \\
\text { (R eye) }\end{array}$ & $\begin{array}{l}\text { Recent onset; } \\
\text { blood count and } \\
\text { ESR normal }\end{array}$ & $\begin{array}{l}\text { Localized } \\
\text { raised mass }\end{array}$ & $\begin{array}{l}\text { A sheet of lymphocytes } \\
\text { with ill-defined germinal } \\
\text { follicles and occasional } \\
\text { plasma cells and } \\
\text { reticulum cells }\end{array}$ & Biopsy & Nil & $\begin{array}{l}\text { Alive and well } 7 \\
\text { years after onset; } \\
\text { no tumour } \\
\text { present }\end{array}$ \\
\hline 21 & 62 & $\mathbf{M}$ & $\begin{array}{l}\text { Lower } \\
\text { fornix } \\
\text { (L eye) }\end{array}$ & $\begin{array}{l}\text { Recent onset of } \\
\text { lesions of the } \\
\text { skin and cervical } \\
\text { lymph nodes }\end{array}$ & $\begin{array}{l}\text { Localized } \\
\text { raised mass }\end{array}$ & $\begin{array}{l}\text { A sheet of lymphocytes; } \\
\text { epithelium infiltrated } \\
\text { some fibrosis present }\end{array}$ & $\begin{array}{l}\text { Biopsy and } \\
\text { irradiation }\end{array}$ & Nil & $\begin{array}{l}\text { Died } 4 \text { years } \\
\text { after onset with } \\
\text { metastases in the } \\
\text { spleen, liver, and } \\
\text { lymph nodes }\end{array}$ \\
\hline 22 & 58 & $\mathbf{M}$ & $\begin{array}{l}\text { Bulb and } \\
\text { inner } \\
\text { canthus } \\
\text { ( } R \text { eye) }\end{array}$ & $\begin{array}{l}\text { Gradual onset } \\
\text { over } 6 \text { months; } \\
\text { blood count and } \\
\text { ESR normal; } \\
\text { lesion on opposite } \\
\text { side recurred } 2 \text { \& } \\
\text { years and } 3 \text { years } \\
\text { after excision }\end{array}$ & $\begin{array}{l}\text { Localized } \\
\text { raised mass }\end{array}$ & $\begin{array}{l}\text { A sheet of lymphocytes } \\
\text { with a few plasma cells } \\
\text { and reticulum cells; } \\
\text { infiltration of lymphatic } \\
\text { vessels }\end{array}$ & Excision & $\begin{array}{l}\text { Six-and-a-half } \\
\text { years after } \\
\text { onset }\end{array}$ & $\begin{array}{l}\text { Alive and well } \\
6 \frac{1}{2} \text { years after } \\
\text { onset; residual } \\
\text { bilateral lesions } \\
\text { at both inner } \\
\text { canthi }\end{array}$ \\
\hline 23 & 51 & $\mathbf{F}$ & $\begin{array}{l}\text { Upper } \\
\text { fornix } \\
\text { (R eye) }\end{array}$ & $\begin{array}{l}\text { Present } 2 \text { years } \\
\text { with ptosis; } \\
\text { blood count and } \\
\text { ESR normal; } \\
\text { similar lesion on } \\
\text { opposite side }\end{array}$ & $\begin{array}{l}\text { Widespread } \\
\text { raised lesion }\end{array}$ & $\begin{array}{l}\text { A sheet of lymphocytes } \\
\text { with a fairly large } \\
\text { number of plasma cells, } \\
\text { a few Russell bodies, } \\
\text { and a few reticulum } \\
\text { cells, some of the } \\
\text { latter being } \\
\text { multilobulated }\end{array}$ & $\begin{array}{l}\text { Biopsy and } \\
\text { irradiation }\end{array}$ & $\begin{array}{l}\text { Three, } 4 \text { and } 6 \\
\text { years after } \\
\text { onset, and } \\
\text { treated by } \\
\text { irradiation }\end{array}$ & $\begin{array}{l}\text { Died } 7 \text { years } \\
\text { after onset with } \\
\text { metastases in the } \\
\text { liver and spleen }\end{array}$ \\
\hline 24 & 40 & $\mathbf{F}$ & $\begin{array}{l}\text { Upper } \\
\text { fornix and } \\
\text { inner } \\
\text { canthus } \\
\text { (R eye) }\end{array}$ & $\begin{array}{l}\text { Present } 4 \text { years; } \\
\text { gradual increase } \\
\text { in size; blood } \\
\text { count and ESR } \\
\text { normal; similar } \\
\text { lesion in opposite } \\
\text { eye recurred } 5 \\
\text { years, } 6 \text { years, and } \\
8 \text { years after } \\
\text { onset, and } \\
\text { irradiated }\end{array}$ & $\begin{array}{l}\text { Widespread } \\
\text { raised lesion }\end{array}$ & $\begin{array}{l}\text { A sheet of lymphocytes } \\
\text { and occasional } \\
\text { reticulum cells }\end{array}$ & $\begin{array}{l}\text { Biopsy and } \\
\text { irradiation } \\
(3000 \mathrm{r} \text { in } 21 \\
\text { days })\end{array}$ & Nil & $\begin{array}{l}\text { Alive and well } 8 \\
\text { years after onset, } \\
\text { with no evidence } \\
\text { of any tumour }\end{array}$ \\
\hline 25 & 50 & $\mathbf{M}$ & $\begin{array}{l}\text { Bulb } \\
\text { (L eye) }\end{array}$ & $\begin{array}{l}\text { Present } 9 \\
\text { months; blood } \\
\text { count normal; } \\
\text { similar lesion } \\
\text { in other eye } \\
\text { irradiated; } \\
\text { hepatomegaly } \\
\text { and inguinal } \\
\text { lymphadenopathy } \\
\text { for same period }\end{array}$ & $\begin{array}{l}\text { Localized } \\
\text { papillary } \\
\text { lesion }\end{array}$ & $\begin{array}{l}\text { A sheet of lymphocytes } \\
\text { with a few reticulum } \\
\text { cells }\end{array}$ & $\begin{array}{l}\text { Biopsy and } \\
\text { irradiation } \\
(750 \mathrm{r} \text { in } 15 \\
\text { days) }\end{array}$ & Nil & $\begin{array}{l}\text { Died } 4 \text { years } \\
\text { after onset; } \\
\text { lesions in } L \text { iliac } \\
\text { fossa }\end{array}$ \\
\hline 26 & 50 & $\mathbf{M}$ & $\begin{array}{l}\text { Lower } \\
\text { fornix and } \\
\text { caruncle } \\
\text { (R eye) }\end{array}$ & $\begin{array}{l}\text { Present } 5 \text { years; } \\
\text { blood count and } \\
\text { ESR normal }\end{array}$ & $\begin{array}{l}\text { Localized } \\
\text { papillary } \\
\text { lesion }\end{array}$ & $\begin{array}{l}\text { A sheet of lymphocytes } \\
\text { with prominent } \\
\text { germinal follicles }\end{array}$ & Excision & Nil & $\begin{array}{l}\text { Alive and well } \\
5 \frac{1}{\text { years after }} \\
\text { onset }\end{array}$ \\
\hline
\end{tabular}

Table I Clinico-pathological features, primary treatment, and follow up cases studied-continued

Multilobulated reticulum cells were also present (Fig. 12) and Russell bodies and plasma cells (Fig. 13) were features of case 23.

A review of the clinical and histological features of the four cases diagnosed initially as lymphosarcoma (cases 11, 15, 19, and 24) and did not disseminate is shown in Table V. A tumour which was diagnosed as lymphosarcoma (case 21) and which disseminated four years after the onset is included for comparison. It will be seen that there were no histological features which might have indicated that case 21 would disseminate and that the other four cases would not, nor was the outcome related to the primary treatment (Table I). The histological features of case 19 (Fig. 14) are identical to those of case 21 (Fig. 15), the latter patient dying with widespread lymphosarcomatous lesions.

A comparison of the 20 cases which were diag- 


\begin{tabular}{|c|c|c|c|}
\hline \multicolumn{2}{|c|}{ Case Initial Diagnosis } & \multirow{2}{*}{$\begin{array}{l}\text { Follow } U p \\
\begin{array}{l}\text { Alive and well } 18 \\
\text { years after onset }\end{array}\end{array}$} & \multirow{2}{*}{$\begin{array}{l}\text { Final Diagnosis } \\
\begin{array}{l}\text { Non-disseminating } \\
\text { tumour }\end{array}\end{array}$} \\
\hline 1 & Benign lymphoma & & \\
\hline 2 & Benign lymphoma & $\begin{array}{l}\text { Alive and well } 15 \\
\text { years after onset }\end{array}$ & $\begin{array}{l}\text { Non-disseminating } \\
\text { tumour }\end{array}$ \\
\hline 3 & Benign lymphoma & $\begin{array}{l}\text { Died from coronary } \\
\text { thrombosis } 11 \text { years } \\
\text { after onset }\end{array}$ & $\begin{array}{l}\text { Non-disseminating } \\
\text { tumour }\end{array}$ \\
\hline 4 & Benign lymphoma & $\begin{array}{l}\text { Alive and well } 16 \\
\text { years after onset }\end{array}$ & $\begin{array}{l}\text { Non-disseminating } \\
\text { tumour }\end{array}$ \\
\hline 5 & Benign lymphoma & $\begin{array}{l}\text { Alive and well } 11 \\
\text { years after onset }\end{array}$ & $\begin{array}{l}\text { Non-disseminating } \\
\text { tumour }\end{array}$ \\
\hline 6 & Benign lymphoma & $\begin{array}{l}\text { Died from pneu- } \\
\text { monia } 5 \text { years after } \\
\text { onset }\end{array}$ & $\begin{array}{l}\text { Non-disseminating } \\
\text { tumour }\end{array}$ \\
\hline 7 & Benign lymphoma & $\begin{array}{l}\text { Alive and well } 12 \\
\text { years after onset }\end{array}$ & $\begin{array}{l}\text { Non-disseminating } \\
\text { tumour }\end{array}$ \\
\hline 8 & Benign lymphoma & $\begin{array}{l}\text { Died from cardiac } \\
\text { failure } 4 \frac{1}{2} \text { years after } \\
\text { onset }\end{array}$ & $\begin{array}{l}\text { Non-disseminating } \\
\text { tumour }\end{array}$ \\
\hline 9 & Benign lymphoma & $\begin{array}{l}\text { Alive and well } 6 \\
\text { years after onset }\end{array}$ & $\begin{array}{l}\text { Non-disseminating } \\
\text { tumour }\end{array}$ \\
\hline 10 & Benign lymphoma & $\begin{array}{l}\text { Alive and well } 8 \\
\text { years after onset }\end{array}$ & $\begin{array}{l}\text { Non-disseminating } \\
\text { tumour }\end{array}$ \\
\hline 11 & Lymphosarcoma & $\begin{array}{l}\text { Alive and well } 10 \\
\text { years after onset }\end{array}$ & $\begin{array}{l}\text { Non-disseminating } \\
\text { tumour }\end{array}$ \\
\hline 12 & Lymphosarcoma & $\begin{array}{l}\text { Died } 1 \text { year after } \\
\text { onset with } \\
\text { widespread } \\
\text { metastases }\end{array}$ & $\begin{array}{l}\text { Disseminating } \\
\text { tumour }\end{array}$ \\
\hline 13 & Benign lymphoma & $\begin{array}{l}\text { Died from coronary } \\
\text { thrombosis } 5 \frac{1}{2} \text { years } \\
\text { after onset }\end{array}$ & $\begin{array}{l}\text { Non-disseminating } \\
\text { tumour }\end{array}$ \\
\hline 14 & Benign lymphoma & $\begin{array}{l}\text { Died from } \\
\text { lymphosarcoma } 5 \\
\text { years after onset }\end{array}$ & $\begin{array}{l}\text { Disseminating } \\
\text { tumour }\end{array}$ \\
\hline 15 & Lymphosarcoma & $\begin{array}{l}\text { Alive and well } 7 \\
\text { years after onset }\end{array}$ & $\begin{array}{l}\text { Non-disseminating } \\
\text { tumour }\end{array}$ \\
\hline 16 & Benign lymphoma & $\begin{array}{l}\text { Alive and well } 13 \\
\text { years after onset }\end{array}$ & $\begin{array}{l}\text { Non-disseminating } \\
\text { tumour }\end{array}$ \\
\hline
\end{tabular}

\begin{tabular}{|c|c|c|c|}
\hline Case & e Initial Diagnosis & Follow Up & Final Diagnosis \\
\hline 17 & Benign lymphoma & $\begin{array}{l}\text { Died from cardiac } \\
\text { failure } 4 \text { years after } \\
\text { onset }\end{array}$ & $\begin{array}{l}\text { Non-disseminating } \\
\text { tumour }\end{array}$ \\
\hline 18 & Lymphosarcoma & $\begin{array}{l}\text { Died } 1 \text { year after } \\
\text { onset }\end{array}$ & $\begin{array}{l}\text { Disseminating } \\
\text { tumour }\end{array}$ \\
\hline 19 & Lymphosarcoma & $\begin{array}{l}\text { Died from nephritis } \\
8 \text { years after onset }\end{array}$ & $\begin{array}{l}\text { Non-disseminating } \\
\text { tumour }\end{array}$ \\
\hline 20 & Benign lymphoma & $\begin{array}{l}\text { Alive and well } 7 \\
\text { years after onset }\end{array}$ & $\begin{array}{l}\text { Non-disseminating } \\
\text { tumour }\end{array}$ \\
\hline 21 & Lymphosarcoma & $\begin{array}{l}\text { Died } 4 \text { years after } \\
\text { onset }\end{array}$ & $\begin{array}{l}\text { Disseminating } \\
\text { tumour }\end{array}$ \\
\hline 22 & Benign lymphoma & $\begin{array}{l}\text { Alive and well } 6 \frac{1}{2} \\
\text { years after onset }\end{array}$ & $\begin{array}{l}\text { Non-disseminating } \\
\text { tumour }\end{array}$ \\
\hline 23 & Benign lymphoma & $\begin{array}{l}\text { Died } 7 \text { years after } \\
\text { onset }\end{array}$ & $\begin{array}{l}\text { Disseminating } \\
\text { tumour }\end{array}$ \\
\hline 24 & Lymphosarcoma & $\begin{array}{l}\text { Alive and well } 8 \\
\text { years after onset }\end{array}$ & $\begin{array}{l}\text { Non-disseminating } \\
\text { tumour }\end{array}$ \\
\hline 25 & Lymphosarcoma & $\begin{array}{l}\text { Died } 4 \text { years after } \\
\text { onset }\end{array}$ & $\begin{array}{l}\text { Disseminating } \\
\text { tumour }\end{array}$ \\
\hline 26 & Benign lymphoma & $\begin{array}{l}\text { Alive and well } 5 \frac{1}{2} \\
\text { years after onset }\end{array}$ & $\begin{array}{l}\text { Non-disseminating } \\
\text { tumour }\end{array}$ \\
\hline
\end{tabular}

Table II Comparison of final diagnosis with initial diagnosis after follow up

\begin{tabular}{|c|c|c|}
\hline \multirow[t]{2}{*}{ Initial Diagnosis } & \multicolumn{2}{|l|}{ Final Diagnosis } \\
\hline & $\begin{array}{l}\text { Non-disseminating } \\
\text { Tumour }\end{array}$ & $\begin{array}{l}\text { Disseminating } \\
\text { Tumour }\end{array}$ \\
\hline $\begin{array}{l}\text { Benign lymphoma } \\
\text { (18 cases) }\end{array}$ & 16 & 2 \\
\hline $\begin{array}{l}\text { Lymphosarcoma } \\
\text { (8 cases) }\end{array}$ & 4 & 4 \\
\hline
\end{tabular}

Table III Summary of final outcome of the tumours

\begin{tabular}{lll}
\hline Case & Clinical Features & Histopathology \\
\hline 14 & $\begin{array}{l}\text { Recent onset of localized nodular mass in }(\mathrm{L}) \text { fornices; } \\
\text { similar lesion in other conjunctiva treated by irradiation; } \\
\text { blood count normal }\end{array}$ & $\begin{array}{l}\text { A sheet of lymphocytes and occasional reticulum cells, plasma } \\
\text { cells, and eosinophils; a focus of reticulum cells and a few } \\
\text { multilobulated reticulum cells present }\end{array}$ \\
$\begin{array}{l}\text { Widespread, raised lesion in }(\mathbf{R}) \text { upper fornix for } 2 \text { years; } \\
\text { blood count and ESR normal; similar lesion on opposite side }\end{array}$ & $\begin{array}{l}\text { A sheet of lymphocytes with a fairly large number of plasma } \\
\text { cells, a few Russell bodies, and a few reticulum cells, some of } \\
\text { the latter being multilobulated }\end{array}$ \\
4 & $\begin{array}{l}\text { Lesion of }(\mathbf{R}) \text { lower fornix inner canthus and palpebra } \\
\text { conjunctiva for } 2 \text { years }\end{array}$ & $\begin{array}{l}\text { A sheet of lymphocytes with a few plasma cells and reticulum } \\
\text { cells, some of the latter being multilobulated }\end{array}$
\end{tabular}

Table IV Two cases diagnosed as benign lymphoma which disseminated and one not disseminating for comparison

\begin{tabular}{lll}
\hline Case & Clinical Features & Histopathology \\
\hline 11 & $\begin{array}{l}\text { Localized papillary mass in (L) bulbar conjunctiva for } 4 \text { years; lesion } \\
\text { recurred and increased in size gradually }\end{array}$ & $\begin{array}{l}\text { A sheet of lymphocytes with occasional plasma cells } \\
\text { and reticulum cells; marked stromal fibrosis }\end{array}$ \\
$\begin{array}{l}\text { Widespread papillary lesion of }(\mathrm{R}) \text { fornix for } 5 \text { months; gradual } \\
\text { increase in size; similar lesion in other eye irradiated; blood count }\end{array}$ & $\begin{array}{l}\text { A sheet of lymphocytes with occasional reticulum cells, } \\
\text { eosinophils, and mitotic figures }\end{array}$
\end{tabular}
normal

19 Localized raised lesion of (L) bulbar conjunctiva for 18 months

A sheet of lymphocytes and occasional reticulum cells

24 A widespread raised lesion of the (R) upper fornix and inner canthus,

A sheet of lymphocytes and occasional reticulum cells present for 4 years and increasing in size gradually; similar lesion in opposite eye recurred on three occasions; blood count and ESR normal

21 Recent onset of localized raised mass in (L) lower fornix; recent onset of lesions in the skin and cervical lymphadenopathy

A sheet of lymphocytes

Table V Four cases diagnosed as lymphosarcoma which did not disseminate and one disseminating for comparison 


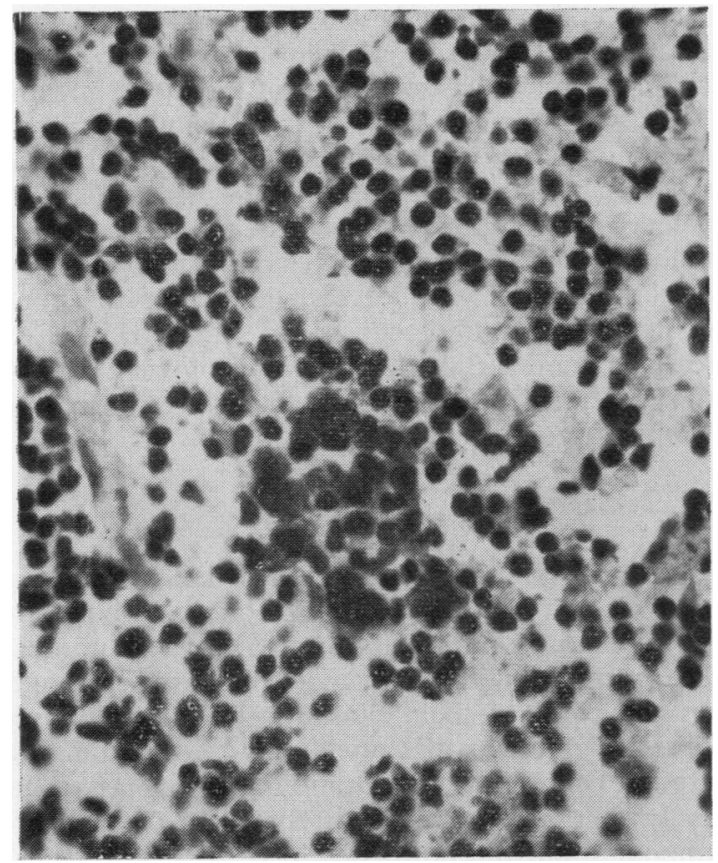

Fig. 11 Lymphocytic tumour (lymphosarcoma) of the conjunctiva which disseminated, showing a sheet of lymphocytes with occasional reticulum cells. $H$ and $E$ $\times 130$.

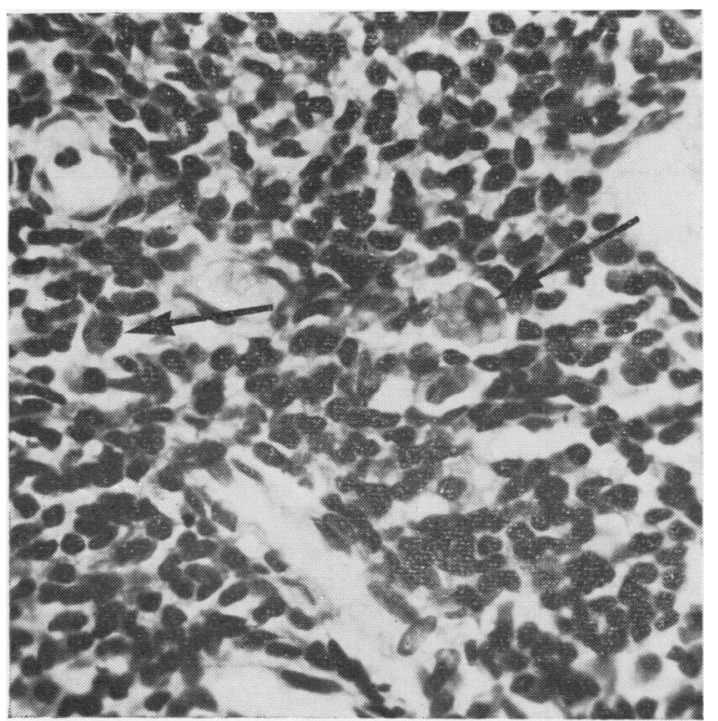

Fig. 13 Lymphocytic tumour (lymphosarcoma) of the conjunctiva, showing plasma cells and Russell bodies (arrows). $H$ and $E \times 590$.

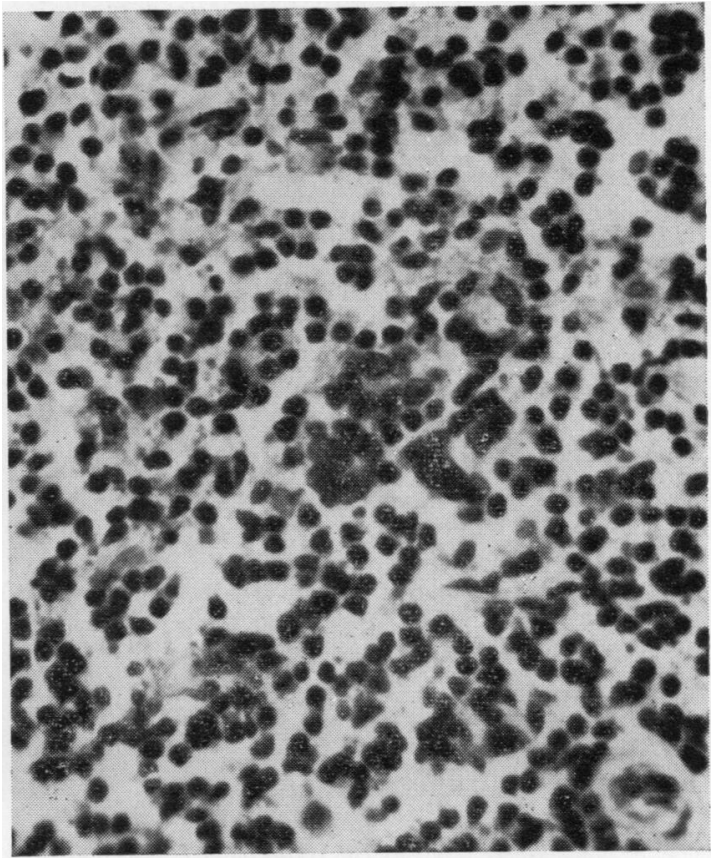

Fig. 12 Lymphocytic tumour (lymphosarcoma) of the conjunctiva, showing multilobulated reticulum cells. $H$ and $E \times 590$.

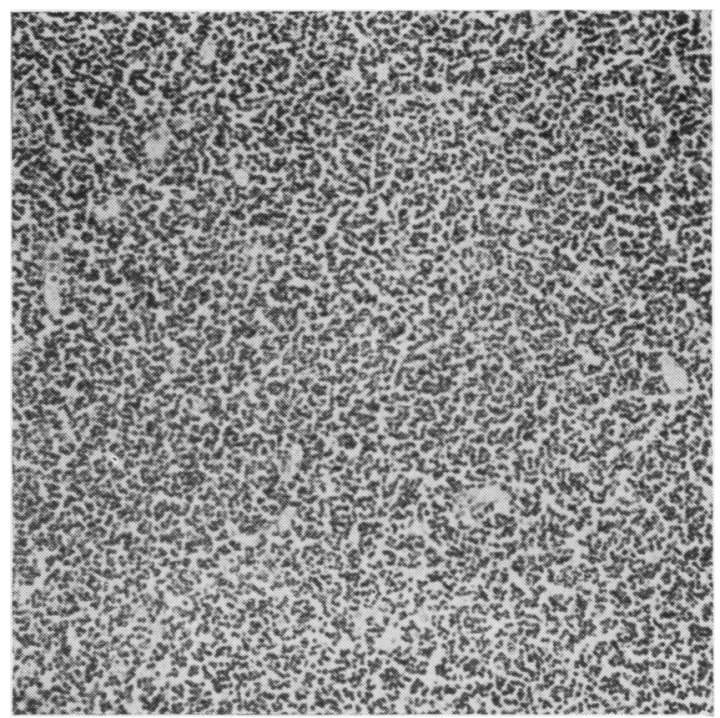

Fig. 14 Lymphocytic tumour (benign lymphoma), showing a sheet of lymphocytes. $H$ and $E \times 80$. 


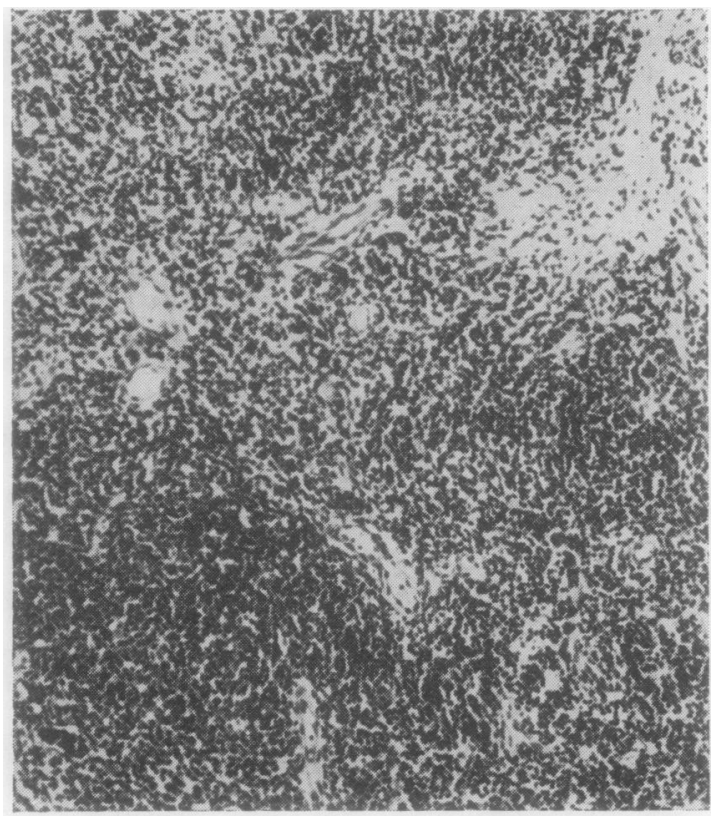

Fig. 15 Lymphocytic tumour (lymphosarcoma) showing $a$ sheet of lymphocytes. $H$ and $E \times 130$.

nosed finally as non-disseminating tumours with the six cases of disseminating tumour is shown in Table VI. It will be seen that there were no distinguishing clinical features between the two groups. As far as the six tumours that disseminated are concerned, I made enquiries about these by letter and can confirm $\frac{0}{\vec{\sigma}}$ that none of them showed any abnormal lymphocytic or lymphoblastic activity in the blood stream. $\overrightarrow{\bar{B}}$ Two tumours (cases 20 and 26) in the non-dissemi- $\frac{\overrightarrow{0}}{0}$ nating group showed germinal follicles which were $\frac{\bar{\sigma}}{\circ}$ not seen in any of the six tumours which dissemi- $\bar{\omega}$ nated. One tumour that disseminated showed $\vec{\nabla}$ infiltration of the tissues by lymphoblasts (case 12), a feature which was not seen in any of the non- $\omega$ disseminating tumours. Apart from these two $\overrightarrow{0}$ features, however, there were no histological criteria $\overrightarrow{-}$ to suggest a diagnosis of either benign lymphoma or $\vec{\omega}$ lymphosarcoma.

\section{Discussion}

Twenty-six lymphocytic tumours of the conjunctiva of were classified into benign lymphoma and lympho-o sarcoma on their initial histological appearances. $\stackrel{?}{-}$ They were followed up for more than five years, and $\vec{O}$ on the basis of their clinical behaviour were re- $?$ classified into non-disseminating and disseminating음 groups. Of the 18 'benign' tumours, two metastasized $\stackrel{\Phi}{?}$ as lymphosarcomas and of the eight 'malignant' $\vec{\omega}$ tumours, four did not disseminate. The histological $\triangle$ features of these two groups are very similar or identical apart from the fact that one tumour (case? 12, a lymphosarcoma) showed lymphoblastic infiltration of the conjunctiva and that germina follicles were found only in those tumours which dido not disseminate. Apart from these two histological features there are no means by which the pathologisto

No. of Cases Histology Non-disseminating Disseminating

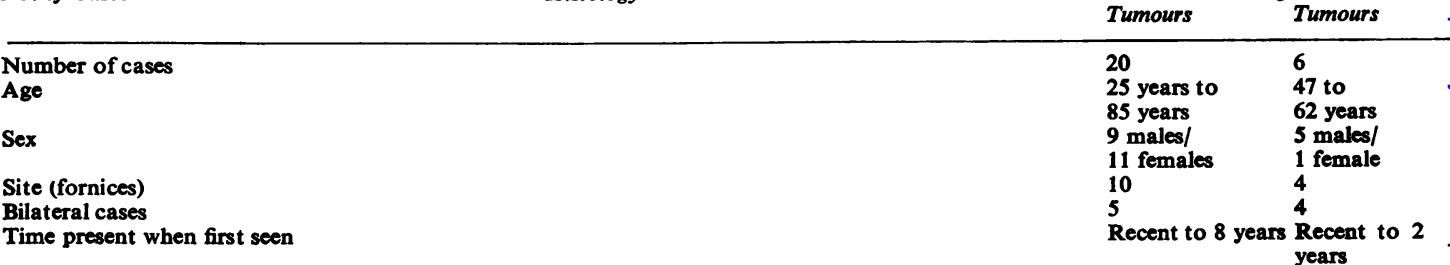

Rapid growth of lesion (when recorded) Slow growth of lesion (when recorded)

Localized growth of lesion (when recorded)

Widespread growth of lesion (when recorded)

Normal blood count (when done)

Normal ESR (when done)

Raised ESR (when done)

Other inflammatory cells present

Multilobulated reticulum cells present Foci of reticulum cells present

Germinal follicles present

Infiltration of tissues by lymphoblasts

Infiltration of lymphatic vessels by lymphocytes

Infiltration of epithelium by lymphocytes

Mitotic figures

Fibrosis years

0

1

Table VI A clinico-pathological comparison of 20 non-disseminating and six disseminating tumours 
can foretell the outcome of these tumours, and a prolonged follow up is necessary in order to arrive at a diagnosis of benignity or malignancy.

The nature of these non-disseminating lymphocytic tumours is not known. It is possible that they represent lesions of lymphoid hyperplasia due to some aetiological agent such as a microorganism, chemical injury, or irradiation. The presence of other inflammatory cells and germinal follicles in some cases support this contention, but no evidence of any aetiological agent has been demonstrated in any of the reported cases. The other possibility is that they may be true neoplasms having a very low degree of malignant potential, and this suggestion is supported by the fact that they have a similar or identical histological picture to lymphosarcoma. If they are malignant tumours, however, it is strange that they do not eventually disseminate even after prolonged periods of time. At the present moment the question of their true nature must remain unanswered.

I am most grateful to Professor Norman Ashton for his advice during the preparation of this article.

\section{References}

Ayoub, H. (1948). Conjunctival lymphoid hyperplasia. Bull. ophthal. Soc. Egypt, 38, 91-92.

Baslini, C. (1907). Contributo allo studio clinico e anatomico del linfoma della congiuntiva e del tarso. Clin. oculist., 8, 28502853.

Bedell, A. J. (1922). So called solid edema; lymphoma. Amer. J. Ophthal., 5, 526-530.

Bernardi, L., Martuzzi M., and Palmieri, L. (1963). La neoplasie reticulo-istiocitarie primitive della congiuntiva. G. ital. Oftal., 16, $132-159$.

Blaxter, P. L. (1955). Conjunctival lymphoma treated by X-ray. Trans. ophthal. Soc. U.K., 75, 315-320.

Buiuc, S., Cordun, G., Beșchea, B., Popovici, E., and Rinea, M. (1966). Unilateral palbebro-orbital lymphoma. Oftalmologia (Buc.), 10, 169-172.

Capalbi, S. (1951). Neoplasia maligna del connettivo-reticolare della congiuntiva. Atti Soc. oftal. Lombarda, 6, 184

Casolino, L. (1916). Di un linfo-sarcoma della congiuntiva bulbare. Arch. Ottal, 23, 383-398.

Charamis, J. (1957). Lymphoma of the conjunctiva. Bull. Soc. hell in. Ophtal., 25, 45-47.

Chatterjee, B. M. (1959). Subconjunctival lymphoma. Brit.J. Ophthal., 43, 371-375.

Coats, G. (1915). Lymphoma and lymphosarcoma of the conjunctiva. Arch. Ophthal., 44, 235-245.

Cometta, F. (1952). Lymphome ou lymphosarcome de la conjonctive? Arch. Ophtal. (Paris), 12, 405-414.
Cosmettatos, G. F. (1910). Lymphom der Bindehaut. Arch. Augenheilk., 67, 391-396.

Duyse, D. van (1905). Prolifération lymphomateuse hyaline de la conjonctive bulbaire droite. Arch. Ophtal. (Paris), 25, 402-421.

Ennema, M. C. (1935). Lymphoma conjunctivae. Acta ophthal. (Kbh.), $13,225-239$.

Fergus, A. F. (1905). Malignant disease of conjunctiva. Trans. ophthal. Soc. U.K., 25, 40-46.

Friedman, B., Borrelli, F. J., and Geleris, I. (1955). Lymphosarcoma of the bulbar conjunctiva. Arch. Ophthal., 54, 381-385.

Fronimopoulos, J., Kofinas, H., and Lambrou, N. (1964). A case of conjunctival lymphoma. Bull. Soc. hellén. Ophtal., 32, 12-14.

Goldzieher, W. (1907). Ein Fall von hämorrhagischer Adenie mit symmetrischen Lymphomen der Bindehaut. Albrecht $V$. Graefes Arch. Ophthal., 67, 71-77.

Heath, P. (1949). Ocular lymphomas. Amer. J. Ophthal., 32, 12131223.

Heritier, F., and Wachtel, L. (1951). Lymphosarcomatose viscérale a localisation colique et oculaire. J. Radiol. Électrol., 32, 790793.

Hilaris, G., Katrakis, G., and Karapatakis, D. (1959). Epibulbar lymphoma. Bull. Soc. hellén. Ophtal., 26, 182-183.

Hochheim, W. (1900). Ein Beitrag zur Kenntniss der symmetrischen Lid und Orbitaltumoren. Albrecht v. Graefes Arch. Ophthal., $51,347-379$.

Junghannes, K. (1953). Beitrag zur Retothelsarkomatose am Auge. Klin. Mbl. Augenheilk., 123, 95-98.

King, E. F., and Ashton, N. (1949). A case of lymphoma of the conjunctiva. Trans. ophthal. Soc. U.K., 69, 301-307.

Lerman, S. (1960). Primary lymphosarcoma of the conjunctiva. Amer. J. Ophthal., 50, 147-148.

Magni, S. (1949). Reticulo-istiocitomi della congiuntiva. G. ital. Oftal., 2, 427-445.

Offret, G., and Rouher, F. (1956). Réticulose ou réticulo-lymphosarcome de la conjonctive. Bull. Soc. Ophtal. Fr., pp. 478483.

Roveda, J. M. (1949). Linfosarcoma de la conjuntiva. Arch. Oftal. B. Aires, 24, 247-253.

Saradarian, A. V. (1940). Bilateral subconjunctival lymphoid infiltration. Arch. Ophthal., 24, 980-983.

Schardt, W. M. (1956). Lymphosarcoma of the conjunctiva; a case report. Conn. med. J, . 20, 199.

Scuderi, G., and Cardia, L. (1959). Contributo allo studio delle neoplasie reticulo-endoteliali della congiuntiva. Boll. Oculist., 38, 616-628.

Shannon, C. E. G., and McAndrews, L. F. (1932). Lymphomas of the conjunctiva. Amer. J. Ophthal., 15, 821-825.

Speciale-Piccichè, P. (1927). Linfoma della congiuntiva. Ann. Ottal. $55,412-418$.

Svoboda, J. (1948). Lymfom spojivky. Čs. Oftal., 4, 296-300.

Teulières, M. (1910). La lymphome de la conjonctive. Bull. Soc. franc. Ophtal., pp. 548-553.

Theodore, F. H. (1960). The lids, lacrimal apparatus, and conjunctiva. Arch. Ophthal., 63, 160-194.

Trzcińska-Dąbrowska, Z. (1963). Two cases of lymphoma of the conjunctiva. Klin. oczna, 33, 191-196.

Valvo, G. (1959). Infeltrazione linfomatosa della congiuntiva. Arch. Ottal., 63, 345-355.

Vancea, P., Lazarescu, D., Cerne, G., and Vaighel, V. (1959). Sarcome primitif de la conjonctive généralisé à la totalité de l'appareil lymphatique. Arch. Ophtal. (Paris), 19, 5-12.

Vollara, A. L. (1899). Linfosarcoma della plica semilunare. Ann. Ottal., 28, 58-68.

Werner, L. (1904). Lymphoma of the conjunctiva. Trans. ophthal. Soc U.K., 24, 24-30. 